Mathematical Modelling and Analysis

Volume 19 Number 2, April 2014, 180-198

http://dx.doi.org/10.3846/13926292.2014.909897

(C) Vilnius Gediminas Technical University, 2014
Publisher: Taylor\&Francis and VGTU

http://www.tandfonline.com/TMMA

Print ISSN: 1392-6292

Online ISSN: 1648-3510

\title{
A Reproducing Kernel Method for Solving a Class of Nonlinear Systems of PDEs
}

\section{Maryam Mohammadi and Reza Mokhtari}

\author{
Isfahan University of Technology \\ 84156-83111 Isfahan, Iran \\ E-mail: m_mohammadi@math.iut.ac.ir \\ E-mail(corresp.): mokhtari@cc.iut.ac.ir
}

Received May 4, 2013; revised March 10, 2014; published online April 15, 2014

\begin{abstract}
This paper is concerned with a technique for solving a class of nonlinear systems of partial differential equations (PDEs) in the reproducing kernel Hilbert space. The analytical solution is represented in the form of series. An iterative method is given to obtain the approximate solution. The convergence analysis is established theoretically. The proposed method is successfully used for solving a coupled system of viscous Burgers' equations and a nonlinear hyperbolic system. Performance of the method is tested in terms of various error norms. In the case of non-availability of exact solution, it is compared with the existing methods.
\end{abstract}

Keywords: reproducing kernel space, coupled viscous Burgers' equations, nonlinear hyperbolic system.

AMS Subject Classification: 65M99; 35C10.

\section{Introduction}

A large number of problems in science and engineering such as problems posed in solid state physics, fluid mechanics, chemical physics, plasma physics, optics and etc, are modelled as nonlinear PDEs or systems of nonlinear PDEs, see e.g. $[5,7,9,14,15]$ and references cited therein.

Nonlinear systems of PDEs have attracted much attention in studying evolution equations. Many authors have studied the analytical and approximate solutions of nonlinear systems of PDEs by using various techniques. Some of them are the methods of exp-function [13], Chebyshev spectral collocation [9], radial basis functions collocation [7], compactly supported radial basis functions collocation [20], Chebyshev pseudospectral multidomain [5], cubic B-spline collocation [10], and Fourier pseudospectral [18].

The theory of reproducing kernels [1], was used for the first time at the beginning of the 20th century by S. Zaremba in his work on boundary value problems for harmonic and biharmonic functions. This theory has been suc- 
cessfully applied for solving a bunch of problems, see e.g. $[3,6,11,12,16]$ and references cited therein.

In this study, a general technique is proposed in the reproducing kernel space for solving the following class of nonlinear systems of PDEs:

$$
\left\{\begin{array}{l}
L_{1}\left(u_{1}(x, t)\right)=N_{1}(x, t, U(x, t))+F_{1}(x, t), \\
\cdots \\
L_{k}\left(u_{k}(x, t)\right)=N_{k}(x, t, U(x, t))+F_{k}(x, t),
\end{array} \quad(x, t) \in \Omega=(a, b) \times(0, T]\right.
$$

with the initial and boundary conditions

$$
\begin{aligned}
& u_{i}(x, 0)=0, \quad x \in[a, b], \\
& u_{i}(a, t)=0, \quad u_{i}(b, t)=0, \quad t \in[0, T],
\end{aligned}
$$

where for $i=1, \ldots, k, L_{i}$ 's and $N_{i}$ 's are linear and nonlinear differential operators, respectively, $F_{i}(x, t)$ are given functions and $U(x, t)=\left[u_{1}(x, t), u_{2}(x, t)\right.$, $\left.\ldots, u_{k}(x, t)\right]^{T}$ is an unknown vector function to be determined. We assume that (1.1) is of one-order derivative in $t$, and (1.1)-(1.3) has a unique solution. we only consider the homogeneous initial and boundary conditions, because the non-homogeneous initial and boundary conditions can be easily transformed to the homogeneous ones. If we construct a function space, in which each function $u_{i}(x, t)$ satisfies (1.2)-(1.3), we can solve (1.1) in the function space.

The advantages of the approach lie in the following facts. The method is mesh free, easily implemented and capable in treating various boundary conditions. The method needs no time discretization against $[7,10]$ and any ODE integrator against $[5,9,18]$. Therefore there is no concern about the stability problem and also increasing the end of time $T$ does not increase the CPU time. Also we can evaluate the approximate solution $U_{n, m}(x, t)$ for fixed $n$ and $m$ once, and use it over and over. The approximate solution also converges uniformly to the analytical solution. Unlike the previous studies on the reproducing kernel methods, we avoid having to practise the Gram-Schmidt orthogonalization process. Then the required computational time can be somewhat saved.

The rest of the paper is organized as follows. In Section 2 a brief introduction of the reproducing kernel Hilbert spaces are represented. The problem solving, method implementation and verification of convergence of the approximate solution to the analytical solution are prepared in Sections 3-4. In Section 5, we define several reproducing kernel spaces. Numerical results are presented in Section 6. The last section is devoted to a brief conclusion.

\section{Preliminaries}

We start with some basic definitions which play a very important role in the study of reproducing kernel Hilbert spaces [1].

Definition 1 . Let $H$ be a Hilbert space of functions $f: \Omega \rightarrow \mathbb{C}$. Denote by $\langle\cdot, \cdot\rangle$ the inner product and let $\|\cdot\|=\sqrt{\langle\cdot, \cdot\rangle}$ be the induced norm in $H$. 
The function $K(x, y): \Omega \times \Omega \rightarrow \mathbb{C}$ is called a reproducing kernel of $H$ if the followings are satisfied:

(i) $K_{y}(x)=K(x, y) \in H \quad$ for all $y \in \Omega$,

(ii) $f(y)=\left\langle f(x), K_{y}(x)\right\rangle$ for all $f \in H$ and for all $y \in \Omega$.

Definition 2. A Hilbert space $H$ of functions on a set $\Omega$ is called a reproducing kernel Hilbert space if there exists a reproducing kernel $K$ of $H$.

Remark 1. The existence of the reproducing kernel of a Hilbert space $H$ is due to the Riesz Representation Theorem. It is known that the reproducing kernel is unique.

Definition 3. Let $K: \Omega \times \Omega \rightarrow \mathbb{C}$ be a kernel on $\Omega$. The kernel $K$ is called Hermitian if for any set of points $\left\{y_{1}, \ldots, y_{n}\right\} \subseteq \Omega$ and any complex numbers $\epsilon_{1}, \ldots, \epsilon_{n}$ we have $\sum_{i, j=1}^{n} \epsilon_{i} \bar{\epsilon}_{j} K\left(y_{i}, y_{j}\right) \in \mathbb{R}$, and $K$ is called positive definite if $\sum_{i, j=1}^{n} \epsilon_{i} \bar{\epsilon}_{j} K\left(y_{i}, y_{j}\right) \geq 0$.

Theorem 1. The reproducing kernel $K_{y}(x)$ of a reproducing kernel Hilbert space $H$ is positive definite.

It has been proved that to every positive definite kernel $K$ corresponds one and only one class of functions $F$ with a uniquely determined inner product in it, forming a Hilbert space and admitting $K$ as a reproducing kernel. In fact the kernel $K$ produces the entire space $H$, i.e.,

$$
H=\overline{\operatorname{span}\{K(z, \cdot) \mid z \in \Omega\}} \text {. }
$$

We note that it is possible to define several different inner products in the same class of functions $H$, so that $H$ is complete with respect to each one of the corresponding norms. To each one of the Hilbert space $(H,\langle\cdot, \cdot\rangle)$ there corresponds one and only one kernel function $K$. Therefore, $K$ depends not only on the class of functions in $H$, but also on the choice of the inner product that $H$ admits.

\section{The Method Implementation}

Consider one of the equations of (1.1), as an example

$$
L_{1}\left(u_{1}(x, t)\right)=N_{1}(x, t, U(x, t))+F_{1}(x, t),
$$

where $L_{1}: W(\Omega) \rightarrow \bar{W}(\Omega)$ is an invertible bounded linear differential operator, $N_{1}$ is a nonlinear differential operator, $F_{1}(x, t)$ is an arbitrary function and $U(x, t)=\left[u_{1}(x, t), u_{2}(x, t), \ldots, u_{k}(x, t)\right]^{T}$. The spaces $W(\Omega)$ and $\bar{W}(\Omega)$ are reproducing kernel spaces which are defined according to the highest derivatives of (1.1) and the initial and boundary conditions (1.2)-(1.3). Let $K_{(y, s)}(x, t)$ and $\bar{K}_{(y, s)}(x, t)$ be the reproducing kernels of $W(\Omega)$ and $\bar{W}(\Omega)$, respectively. We choose a countable dense subset $\left\{\left(x_{j}, t_{j}\right)\right\}_{j=1}^{\infty}$ in $\bar{\Omega}$, and define

$$
\phi_{j}(x, t)=\bar{K}_{\left(x_{j}, t_{j}\right)}(x, t), \quad \psi_{j 1}(x, t)=L_{1}^{*} \phi_{j}(x, t),
$$


where $L_{1}^{*}$ is the adjoint operator of $L_{1}$. It can be shown that [11]

$$
\psi_{j 1}(x, t)=\left.L_{1(y, s)} K_{(y, s)}(x, t)\right|_{(y, s)=\left(x_{j}, t_{j}\right)} .
$$

The subscript $(y, s)$ by the operator $L_{1}$ indicates that the operator $L_{1}$ applies to the function of $(y, s)$.

Theorem 2. Suppose that $\left\{\left(x_{j}, t_{j}\right)\right\}_{j=1}^{\infty}$ is dense in $\bar{\Omega}$, then the analytical solution of (3.1) can be represented as

$$
u_{1}(x, t)=\sum_{j=1}^{\infty} a_{j 1} \psi_{j 1}(x, t),
$$

where the coefficients $a_{j}$ are determined by solving the following semi-infinite system of linear equations

$$
\mathbf{B}_{1} \mathbf{a}_{1}=\mathbf{N}_{1}
$$

in which

$$
\begin{aligned}
& \mathbf{B}_{1}=\left[\left.L_{1} \psi_{j 1}(x, t)\right|_{(x, t)=\left(x_{i}, t_{i}\right)}\right]_{i, j=1,2, \ldots}, \quad \mathbf{a}_{1}=\left[a_{11}, a_{21}, \ldots\right]^{T}, \\
& \mathbf{N}_{1}=\left[N_{1}\left(x_{1}, t_{1}, U\left(x_{1}, t_{1}\right)\right)+F_{1}\left(x_{1}, t_{1}\right), N_{1}\left(x_{2}, t_{2}, U\left(x_{2}, t_{2}\right)\right)+F_{1}\left(x_{2}, t_{2}\right), \ldots\right]^{T} .
\end{aligned}
$$

Proof. Since $\left\{\left(x_{j}, t_{j}\right)\right\}_{j=1}^{\infty}$ is dense in $\bar{\Omega}$, then $\psi_{j 1}(x, t)$ is a complete system in $W(\Omega)$ [11]. So the analytical solution can be represented as (3.2). Since

$$
\begin{aligned}
\left\langle\psi_{i 1}(x, t), \psi_{j 1}(x, t)\right\rangle_{W} & =\left\langle L_{1}^{*} \phi_{i}(x, t), \psi_{j 1}(x, t)\right\rangle_{W} \\
& =\left\langle\phi_{i}(x, t), L_{1} \psi_{j 1}(x, t)\right\rangle_{\bar{W}}=\left.L_{1} \psi_{j 1}(x, t)\right|_{(x, t)=\left(x_{i}, t_{i}\right)}, \\
\left\langle u_{1}(x, t), \psi_{i 1}(x, t)\right\rangle_{W} & =\left\langle u_{1}(x, t), L_{1}^{*} \phi_{i}(x, t)\right\rangle_{W}=\left\langle L_{1} u_{1}(x, t), \phi_{i}(x, t)\right\rangle_{\bar{W}} \\
& =N_{1}\left(x_{i}, t_{i}, U\left(x_{i}, t_{i}\right)\right)+F_{1}\left(x_{i}, t_{i}\right),
\end{aligned}
$$

according to the best approximation in Hilbert spaces [4], the coefficients $a_{j 1}$ are determined by (3.3).

If equations in (1.1) are linear, i.e., $N_{d}(x, t, U(x, t))=0$ for $d=1, \ldots, k$, the analytical solution of each equation can be obtained directly from (3.2) and the approximate solution of each equation is the $m$-term intercept of the analytical solution which can be determined by solving a $m \times m$ system of linear equations, otherwise, we need to construct an iterative method for solving (3.3). For this purpose, we choose the number of points $m$, the number of iterations $n$ and put the initial vector function $U_{0, m}(x, t)=[0,0, \ldots, 0]^{T}$. Then the approximate solution of (1.1) is defined by

$$
U_{n, m}(x, t)=\left[u_{n, m, 1}(x, t), u_{n, m, 2}(x, t), \ldots, u_{n, m, k}(x, t)\right]^{T},
$$

where

$$
\left\{\begin{array}{l}
u_{n, m, 1}(x, t)=\sum_{j=1}^{m} a_{j 1} \psi_{j 1}(x, t), \\
\cdots \\
u_{n, m, k}(x, t)=\sum_{j=1}^{m} a_{j k} \psi_{j k}(x, t),
\end{array}\right.
$$


in which $\psi_{j d}(x, t)$ for $d=1, \ldots, k$ can be defined for each equation similar to the sample equation (3.1), and the coefficients $a_{j d}$ for $d=1, \ldots, k$ are determined by

$$
\begin{aligned}
& \left.\sum_{j=1}^{m} a_{j d} L_{d} \psi_{j d}(x, t)\right|_{(x, t)=\left(x_{i}, t_{i}\right)} \\
& \quad=N_{d}\left(x_{i}, t_{i}, U_{n-1, m}\left(x_{i}, t_{i}\right)\right)+F_{d}\left(x_{i}, t_{i}\right), \quad i=1, \ldots, m .
\end{aligned}
$$

Remark 2. There exist a unique solution for equations (3.5) due to the strictly positive definiteness property of the reproducing kernel.

The results of this section can be summarized in the following algorithm.

\section{Algorithm.}

1. Choose $m$ collocation points in the domain set $\bar{\Omega}$.

2. Set $\psi_{j d}(x, t)=\left.L_{d(y, s)} K_{(y, s)}(x, t)\right|_{(y, s)=\left(x_{j}, t_{j}\right)} ; d=1, \ldots, k, j=1, \ldots, m$.

3. Set $\mathbf{B}_{d}=\left[\left.L_{d} \psi_{j d}(x, t)\right|_{(x, t)=\left(x_{i}, t_{i}\right)}\right]_{i, j=1,2, \ldots, m} ; d=1, \ldots, k$.

4. Choose the number of iterations $n$.

5. Set $i=0$.

6. Set the initial vector function $U_{i, m}(x, t)=[0,0, \ldots, 0]^{T}$.

7. Set $i=i+1$.

8. For $d=1, \ldots, k$ set $\mathbf{N}_{d}=\left[N_{d}\left(x_{i}, t_{i}, U_{i-1, m}\left(x_{i}, t_{i}\right)\right)+F_{d}\left(x_{i}, t_{i}\right)\right]_{i=1, \ldots, m}^{T}$.

9. For $d=1, \ldots, k$ solve system $\mathbf{B}_{d} \mathbf{a}_{d}=\mathbf{N}_{d}$.

10. For $d=1, \ldots, k$ set $u_{i, m, d}(x, t)=\sum_{j=1}^{m} a_{j d} \psi_{j d}(x, t)$.

11. Set $U_{i, m}(x, t)=\left[u_{i, m, 1}(x, t), u_{i, m, 2}(x, t), \ldots, u_{i, m, k}(x, t)\right]^{T}$.

12. If $i<n$ then go to step 7 else stop the algorithm.

\section{Convergence Analysis}

In this section, we verify that the approximate solution $U_{n, m}(x, t)$ converges to the analytical solution $U(x, t)$, uniformly. At first, the following lemma is given.

Lemma 1. For a positive constant $\gamma, A=\left\{u(x, t) \mid\|u(x, t)\|_{W} \leq \gamma\right\}$ is a compact set in the space $C(\Omega)$ provided that

$$
\left\|\frac{\partial K_{(x, t)}(y, s)}{\partial x}\right\|_{W} \leq c_{1}, \quad\left\|\frac{\partial K_{(x, t)}(y, s)}{\partial t}\right\|_{W} \leq c_{2}
$$

where $c_{1}$ and $c_{2}$ are constants. 
Proof. According to the Arzela-Ascoli Theorem, it is enough to show that $A$ is a bounded and equicontinuous set. Since

$$
\begin{aligned}
K_{(y, s)}(x, t) & =\left\langle K_{(y, s)}(\xi, \eta), K_{(x, t)}(\xi, \eta)\right\rangle_{W} \\
& =\left\langle K_{(x, t)}(\xi, \eta), K_{(y, s)}(\xi, \eta)\right\rangle_{W}=K_{(x, t)}(y, s), \\
\left\|K_{(x, t)}(y, s)\right\|_{W} & =\left\langle K_{(x, t)}(y, s), K_{(x, t)}(y, s)\right\rangle_{W}=K_{(x, t)}(x, t)<\gamma_{1} .
\end{aligned}
$$

For any $u(x, t) \in A$, we have

$$
|u(x, t)|=\left|\left\langle u(y, s), K_{(x, t)}(y, s)\right\rangle_{W}\right| \leq\|u(y, s)\|_{W}\left\|K_{(x, t)}(y, s)\right\|_{W} \leq \gamma \gamma_{1},
$$

where $\gamma_{1}$ is a constant. Hence $A$ is a bounded set in the space $C(\Omega)$. On the other hand,

$$
\begin{aligned}
\left|\frac{\partial u(x, t)}{\partial x}\right| & =\left|\left\langle u(y, s), \frac{\partial K_{(x, t)}(y, s)}{\partial x}\right\rangle_{W}\right| \leq\|u\|_{W}\left\|\frac{\partial K_{(x, t)}(y, s)}{\partial x}\right\|_{W} \\
& \leq \gamma\left\|\frac{\partial K_{(x, t)}(y, s)}{\partial x}\right\|_{W}, \\
\left|\frac{\partial u(x, t)}{\partial t}\right| & =\left|\left\langle u(y, s), \frac{\partial K_{(x, t)}(y, s)}{\partial t}\right\rangle_{W}\right| \leq\|u\|_{W}\left\|\frac{\partial K_{(x, t)}(y, s)}{\partial t}\right\|_{W} \\
& \leq \gamma\left\|\frac{\partial K_{(x, t)}(y, s)}{\partial t}\right\|_{W} .
\end{aligned}
$$

Then for any $u(x, t) \in A$ and $\varepsilon>0$,

$$
|u(x+h, t+k)-u(x, t)| \leq|\nabla u(\xi, \eta)| \cdot|(h, k)| \leq \gamma \sqrt{c_{1}^{2}+c_{2}^{2}}|(h, k)|,
$$

where $\xi \in[x, x+h]$ and $\eta \in[t, t+k]$. So there exists $\delta=\frac{\varepsilon}{\gamma \sqrt{c_{1}{ }^{2}+c_{2}{ }^{2}}}$ such that for $|(h, k)|<\delta$, we get

$$
|u(x+h, t+k)-u(x, t)|<\varepsilon .
$$

Hence $A$ is an equicontinuous set in the space $C(\Omega)$.

Theorem 3. If $L_{d}$ is an invertible bounded linear operator and $N_{d}$ is a nonlinear bounded operator, it can be deduced that $\left\{u_{n, m, d}(x, t)\right\}_{n=1}^{\infty}$ is a bounded sequence of functions in $W(\Omega)$ for $d=1, \ldots, k$.

Proof. We get

$$
\begin{aligned}
& \left\|u_{n, m, d}(x, t)\right\|_{W}^{2}=\left\langle u_{n, m, d}(x, t), u_{n, m, d}(x, t)\right\rangle_{W} \\
& =\left\langle\sum_{j=1}^{m} a_{j d} \psi_{j d}(x, t), \sum_{l=1}^{m} a_{l d} \psi_{l d}(x, t)\right\rangle_{W}=\sum_{j=1}^{m} a_{j d}\left\langle\psi_{j d}(x, t), \sum_{l=1}^{m} a_{l d} \psi_{l d}(x, t)\right\rangle_{W} \\
& =\sum_{j=1}^{m} a_{j d}\left\langle\phi_{j d}(x, t), \sum_{l=1}^{m} a_{l d} L \psi_{l d}(x, t)\right\rangle_{W} \\
& =\sum_{j=1}^{m} a_{j d}\left(\left.\sum_{l=1}^{m} a_{l d} L \psi_{l d}(x, t)\right|_{(x, t)=\left(x_{j}, t_{j}\right)}\right)=\mathbf{a}_{d}^{T} \mathbf{B}_{d} \mathbf{a}_{d},
\end{aligned}
$$


where $\mathbf{a}_{d}=\left(a_{j d}, j=1, \ldots, m\right)^{T}$. Now, since

$$
\mathbf{B}_{d}=\left[\left.L_{d} \psi_{j d}(x, t)\right|_{(x, t)=\left(x_{i}, t_{i}\right)}\right]_{i, j=1,2, \ldots, m}, \quad \mathbf{a}_{d}=\mathbf{B}_{d}{ }^{-1} \mathbf{N}_{d},
$$

the assumptions imply that

$$
\left\|u_{n, m, d}(x, t)\right\|_{W} \leq \gamma, \quad d=1, \ldots, k,
$$

where $\gamma$ is a constant.

Theorem 4. Assume that $\left\{\left(x_{i}, t_{i}\right)\right\}_{i=1}^{\infty}$ is dense in $\bar{\Omega}$ and the assumptions of the Theorem 3 and Lemma 1 hold. Then the approximate solution $U_{n, m}(x, t)$ converges to the analytical solution $U(x, t)$.

Proof. For $d=1, \ldots, k$, we have for $j=1, \ldots, m$ and $n=1,2, \ldots$

$$
L_{d}\left(u_{n, m, d}\left(x_{j}, t_{j}\right)\right)=N_{d}\left(x_{j}, t_{j}, U_{n-1, m}\left(x_{j}, t_{j}\right)\right)+F_{d}\left(x_{j}, t_{j}\right) .
$$

According to Lemma 1 , for $d=1, \ldots, k$, there exists a convergent subsequence $\left\{u_{n_{l}, m, d}(x, t)\right\}_{l=1}^{\infty}$ of $\left\{u_{n, m, d}(x, t)\right\}_{n=1}^{\infty}$ such that $u_{n_{l}, m, d}(x, t) \rightarrow u_{d}(x, t)$, uniformly, as $l \rightarrow \infty, m \rightarrow \infty$. Then for $j=1, \ldots, m$ and $n=1,2, \ldots$ we derive

$$
L_{d}\left(u_{n_{l}, m, d}\left(x_{j}, t_{j}\right)\right)=N_{d}\left(x_{j}, t_{j}, U_{n_{l}-1, m}\left(x_{j}, t_{j}\right)\right)+F_{d}\left(x_{j}, t_{j}\right) .
$$

Since the operators $L_{d}$ and $N_{d}$ are both continuous, after taking limit from both sides of $(4.2)$, it can be inferred that $U(x, t)=\left[u_{1}(x, t), \ldots, u_{k}(x, t)\right]^{T}$ is the analytical solution of (1.1). So $U_{n_{l}, m}(x, t)=\left[u_{n_{l}, m, 1}(x, t), \ldots, u_{n_{l}, m, k}(x, t)\right]^{T}$ is the approximate solution of (1.1).

\section{Several Reproducing Kernel Spaces}

In this section, we define some reproducing kernel spaces which are needed in the next section. The corresponding reproducing kernels can be found by the usual technique in many articles in literature (see [11]).

DEFINITION 4. $W_{1}[0, T]=\left\{u(t) \mid u(t)\right.$ and $u^{\prime}(t)$ are absolutely continuous in $[0, T], u^{\prime \prime}(t) \in L^{2}[0, T]$ and $\left.u(0)=0\right\}$. The inner product and the induced norm in $W_{1}[0, T]$ are defined respectively by

$$
\begin{aligned}
& \langle u, v\rangle_{W_{1}}=b_{1} u^{\prime}(0) v^{\prime}(0)+b_{2} \int_{0}^{T} u^{\prime \prime}(t) v^{\prime \prime}(t) d t, \quad u, v \in W_{1}[0, T], \\
& \|u\|_{W_{1}}=\sqrt{\langle u, u\rangle_{W_{1}}}, \quad u \in W_{1}[0, T],
\end{aligned}
$$

where $b_{1}$ and $b_{2}$ are arbitrary positive constants.

The space $W_{1}[0, T]$ is a reproducing kernel space and its reproducing kernel function $r_{s}(t)$ is given by

$$
r_{s}(t)= \begin{cases}\frac{b_{2} t s}{b_{1}}+\frac{1}{2} t^{2} s-\frac{1}{6} t^{3} & t \leq s \\ -\frac{s^{3}}{6}-\frac{-2 b_{2} s t-b_{1} s^{2} t}{2 b_{1}} & t>s\end{cases}
$$


Definition $5 . W_{2}[a, b]=\left\{u(x) \mid u(x), u^{\prime}(x)\right.$ and $u^{\prime \prime}(x)$ are absolutely continuous in $[a, b], u^{(3)}(x) \in L^{2}[a, b]$ and $\left.u(a)=u(b)=0\right\}$. The inner product and the induced norm in $W_{2}[a, b]$ are defined respectively by

$$
\begin{aligned}
& \langle u, v\rangle_{W_{2}}=e_{1} u^{\prime}(a) v^{\prime}(a)+e_{2} \int_{a}^{b} u^{(3)}(x) v^{(3)}(x) d x, \quad u, v \in W_{2}[a, b] \\
& \|u\|_{W_{2}}=\sqrt{\langle u, u\rangle_{W_{2}}}, \quad u \in W_{2}[a, b],
\end{aligned}
$$

where $e_{1}$ and $e_{2}$ are arbitrary positive constants.

The space $W_{2}[a, b]$ is a reproducing kernel space and its reproducing kernel function is $R_{y}(x)$ given in Appendix.

Definition 6. $W(\Omega)=W_{1}[0, T] \otimes W_{2}[a, b]=\left\{u(x, t) \mid \frac{\partial^{3} u}{\partial x^{2} \partial t}\right.$ is completely continuous in $\Omega, \frac{\partial^{5} u}{\partial x^{3} \partial t^{2}} \in L^{2}(\Omega)$ and $\left.u(x, 0)=u(a, t)=u(b, t)=0\right\}$. The inner product and the induced norm in $W(\Omega)$ are defined respectively by

$$
\begin{aligned}
\langle u(x, t), v(x, t)\rangle_{W}= & \sum_{i=0}^{1} \int_{0}^{T}\left[\frac{\partial^{2}}{\partial t^{2}} \frac{\partial^{i}}{\partial x^{i}} u(a, t) \frac{\partial^{2}}{\partial t^{2}} \frac{\partial^{i}}{\partial x^{i}} v(a, t)\right] d t \\
& +\sum_{j=0}^{1}\left\langle\frac{\partial^{j}}{\partial t^{j}} u(x, 0), \frac{\partial^{j}}{\partial t^{j}} v(x, 0)\right\rangle_{W_{2}} \\
& +\int_{0}^{T} \int_{a}^{b}\left[\frac{\partial^{3}}{\partial x^{3}} \frac{\partial^{2}}{\partial t^{2}} u(x, t) \frac{\partial^{3}}{\partial x^{3}} \frac{\partial^{2}}{\partial t^{2}} v(x, t)\right] d x d t
\end{aligned}
$$

and

$$
\|u\|_{W}=\sqrt{\langle u, u\rangle_{W}}, \quad u \in W(\Omega) .
$$

Theorem 5. $W(\Omega)$ is a reproducing kernel space and its reproducing kernel is

$$
K_{(y, s)}(x, t)=R_{y}(x) r_{s}(t),
$$

such that for any $u(x, t) \in W(\Omega)$

$$
u(y, s)=\left\langle u(x, t), K_{(y, s)}(x, t)\right\rangle_{W} .
$$

Proof. See [21].

Remark 3. It is worthy of mention that the free parameters $b_{1}, b_{2}, e_{1}$, and $e_{2}$ which can be selected in order to improve the accuracy of the method, can be chosen as 1 , in most cases.

Remark 4. It can be shown that [3] for the kernel (5.2),

$$
\frac{\partial^{i+j+l+r}}{\partial x^{i} \partial t^{j} \partial y^{l} \partial s^{r}} K_{(y, s)}(x, t) \in L^{2}(\Omega) \quad i+l \leq 5, j+r \leq 3
$$

and

$$
\frac{\partial^{i+j+l+r}}{\partial x^{i} \partial t^{j} \partial y^{l} \partial s^{r}} K_{(y, s)}(x, t), \quad i+l \leq 4, j+r \leq 2
$$

is completely continuous in $\Omega$. Then the relations (4.1) hold, clearly. 


\section{$6 \quad$ Numerical Results}

In this section, we employ the proposed method to establish numerical solutions of two nonlinear systems of PDEs. The results are compared with the exact solutions and some other existing methods to confirm the efficiency of the proposed method. The uniform and Chebyshev set of points in the region $\bar{\Omega}$, are defined by

$$
\begin{aligned}
x_{i} & =a+\frac{b-a}{N_{1}} i, \quad i=0, \ldots, N_{1}, \\
t_{i} & =\frac{T}{N_{2}} i, \quad i=0, \ldots, N_{2}
\end{aligned}
$$

and

$$
\begin{aligned}
x_{i} & =\frac{1}{2}\left((a+b)-(b-a) \cos \frac{i \pi}{N_{1}}\right), \quad i=0, \ldots, N_{1}, \\
t_{i} & =\frac{T}{N_{2}} i, \quad i=0, \ldots, N_{2}
\end{aligned}
$$

respectively, where $N_{1}$ and $N_{2}$ are the number of subdivisions in the spatial and time domains, respectively, and $m=\left(N_{1}+1\right) \times\left(N_{2}+1\right)$. The uniform set of points are used throughout this section unless it is stated to the contrary. We also report norm infinity and the norm of relative errors of the solutions, which are defined by

$$
L_{\infty}=\max _{1 \leq i \leq N_{1}}\left|u_{i}-\bar{u}_{i}\right|, \quad L_{r}=\left(\frac{\sum_{i=1}^{N_{1}}\left(u_{i}-\bar{u}_{i}\right)^{2}}{\sum_{i=1}^{N_{1}} u_{i}^{2}}\right)^{1 / 2},
$$

where $u$ and $\bar{u}$ are the exact and approximate solutions, respectively. The pointwise rate of convergence in space and time are calculated by using the following formulae:

$$
\ln \left(\frac{E\left(N_{1, i+1}\right)}{E\left(N_{1, i}\right)}\right) / \ln \left(\frac{N_{1, i}}{N_{1, i+1}}\right) \text { and } \ln \left(\frac{E\left(N_{2, i+1}\right)}{E\left(N_{2, i}\right)}\right) / \ln \left(\frac{N_{2, i}}{N_{2, i+1}}\right),
$$

where $E\left(N_{1, i}\right)$ and $E\left(N_{2, i}\right)$ are the norm of the relative errors of the solutions with number of spatial points $N_{1, i}$ and number of time points $N_{2, i}$, respectively. The CPU times for a Pentium(R), Dual CPU, $1.60 \mathrm{GHz}$ are also reported to indicate producing the reasonably accurate results with few seconds of CPU time.

\subsection{Coupled viscous Burgers' equations}

Consider the viscous coupled Burgers' equations

$$
\left\{\begin{array}{l}
\frac{\partial u}{\partial t}-\frac{\partial^{2} u}{\partial x^{2}}+\eta u \frac{\partial u}{\partial x}+\alpha \frac{\partial(u v)}{\partial x}=0, \\
\frac{\partial v}{\partial t}-\frac{\partial^{2} v}{\partial x^{2}}+\eta v \frac{\partial v}{\partial x}+\beta \frac{\partial(u v)}{\partial x}=0,
\end{array} \quad(x, t) \in(a, b) \times(0, T]\right.
$$


with the initial conditions

$$
\left\{\begin{array}{l}
u(x, 0)=u_{0}(x), \\
v(x, 0)=v_{0}(x),
\end{array} \quad x \in[a, b]\right.
$$

and boundary conditions

$$
\left\{\begin{array}{l}
(u(a, t), v(a, t))=\left(f_{1}(t), f_{2}(t)\right), \\
(u(b, t), v(b, t))=\left(g_{1}(t), g_{2}(t)\right),
\end{array} \quad t \in[0, T],\right.
$$

where $\eta$ is a real constant, $\alpha$ and $\beta$ are arbitrary constants depending on the system parameters such as Peclet number and Stokes velocity of particles due to gravity and Brownian diffusivity [17], $u_{0}, v_{0}, f_{1}, f_{2}, g_{1}$, and $g_{2}$ are known functions. After homogenizing the initial and boundary conditions, we get the following new problem

$$
\left\{\begin{array}{l}
L_{1}(u(x, t))=N_{1}(x, t, u(x, t), v(x, t))+F_{1}(x, t), \\
L_{2}(v(x, t))=N_{2}(x, t, u(x, t), v(x, t))+F_{2}(x, t),
\end{array} \quad(x, t) \in \Omega=(a, b) \times(0, T]\right.
$$

with the initial conditions

$$
u(x, 0)=v(x, 0)=0, \quad x \in[a, b]
$$

and the boundary conditions

$$
\begin{aligned}
& u(a, t)=u(b, t)=0, \\
& v(a, t)=v(b, t)=0,
\end{aligned} \quad t \in[0, T],
$$

where

$$
\begin{aligned}
& L_{1}(u(x, t))=\frac{\partial u}{\partial t}-\frac{\partial^{2} u}{\partial x^{2}}, \quad L_{2}(v(x, t))=\frac{\partial v}{\partial t}-\frac{\partial^{2} v}{\partial x^{2}} \\
& N_{1}(x, t, u(x, t), v(x, t))=-\eta(u+\tilde{u}) \frac{\partial(u+\tilde{u})}{\partial x}-\alpha \frac{\partial((u+\tilde{u})(v+\tilde{v}))}{\partial x} \\
& N_{2}(x, t, u(x, t), v(x, t))=-\eta(v+\tilde{v}) \frac{\partial(v+\tilde{v})}{\partial x}-\beta \frac{\partial((u+\tilde{u})(v+\tilde{v}))}{\partial x} \\
& F_{1}(x, t)=-\frac{\partial U}{\partial t}+\frac{\partial^{2} u_{0}}{\partial x^{2}}, \quad F_{2}(x, t)=-\frac{\partial V}{\partial t}+\frac{\partial^{2} v_{0}}{\partial x^{2}}
\end{aligned}
$$

in which

$$
\begin{aligned}
& U=\frac{x-b}{a-b} f_{1}(t)+\frac{x-a}{b-a} f_{2}(t), \quad V=\frac{x-b}{a-b} g_{1}(t)+\frac{x-a}{b-a} g_{2}(t), \\
& \tilde{u}=U+u_{0}-U(x, 0), \quad \tilde{v}=V+v_{0}-V(x, 0) .
\end{aligned}
$$

\section{Test problem I)}

In the first experiment, we take $a=-\pi, b=\pi, T=1, \alpha=1, \beta=1$, and $\eta=-2$ with the initial conditions given by

$$
u(x, 0)=v(x, 0)=\sin (x)
$$


Table 1. Errors of $u(x, t)$ for the coupled viscous Burgers' equations (Test problem I).

\begin{tabular}{|c|c|c|c|c|c|c|c|}
\hline \multirow[b]{2}{*}{$T$} & \multicolumn{3}{|c|}{$\begin{array}{l}\text { Method } \\
(n=5, m=220)\end{array}$} & \multicolumn{2}{|c|}{$\begin{array}{l}{[10]} \\
(h=0.01, \Delta t=0.001)\end{array}$} & \multicolumn{2}{|c|}{$\begin{array}{l}{[18]} \\
(h=0.01, \Delta t=0.001)\end{array}$} \\
\hline & $L_{\infty}$ & $L_{r}$ & CPU time & $L_{\infty}$ & $L_{r}$ & $L_{\infty}$ & $L_{r}$ \\
\hline 0.1 & $4.6 E-06$ & $2.5 E-06$ & $243.86 \mathrm{~s}$ & $1.9 E-06$ & $2.1 E-06$ & & \\
\hline 0.5 & $4.8 E-06$ & $8.8 E-06$ & $243.87 \mathrm{~s}$ & $6.2 E-06$ & $1.0 E-05$ & & \\
\hline 1 & $3.7 E-05$ & $1.1 E-05$ & $243.87 \mathrm{~s}$ & $7.6 E-06$ & $2.0 E-05$ & $1.2 E-05$ & $2.9 E-05$ \\
\hline
\end{tabular}

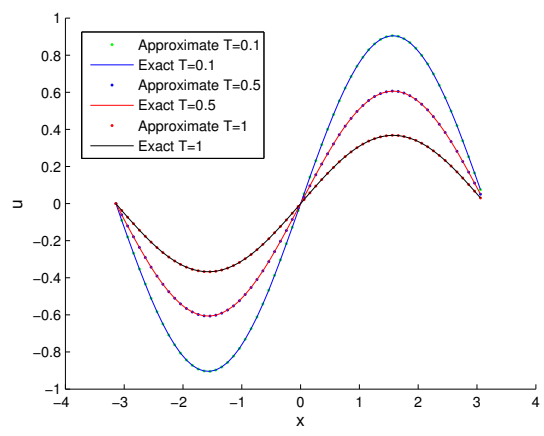

Figure 1. Plots of numerical and exact solutions of the coupled viscous Burgers' equation (Test problem I).

and boundary conditions taken from the exact solution. The exact solution of the equation is given by [8] as $u(x, t)=v(x, t)=\exp (-t) \sin (x)$. In our computational work in this case, we put $e_{1}=1, e_{2}=1, b_{1}=1, b_{2}=1, n=5$, $N_{1}=18$, and $N_{2}=10$. The norm infinity, norm of relative errors, and CPU time of the method for the numerical solution of $u$ are tabulated in Table 1 at different time $T=0.1,0.5,1$. It can be noted from Table 1 that the accuracy of the proposed method are in agreement with [10] and [18]. Numerical solutions of $u$ are also depicted in Figure 1. Absolute error distributions of $u$ at different time for the uniform and Chebyshev points are shown in Figures 2 and 3, respectively. Due to symmetric initial and boundary conditions, results are similar for $v$. In order to examine the point rate of convergence in space and time, computations are carried out with the different number of spatial and time points. In Table 2, the number of time points is kept fixed at $N_{2}=10$ and the number of spatial points $N_{1}=2,4,8,16,32$ is varied to calculate the spatial rate of convergence. In Table 3 , the number of spatial points is kept fixed at $N_{1}=10$ and the number of time points $N_{2}=2,4,8,16,32$ is varied to compute the time rate of convergence. The CPU time corresponding to the different values of $N_{1}$ and $N_{2}$ are also reported in Tables 2 and 3 .

Now, we put

$$
e_{1}=1, \quad b_{1}=1, \quad b_{2}=1, \quad n=5, \quad N_{1}=10, \quad N_{2}=10, \quad T=1 .
$$

Absolute error distributions of $u$ for different values of parameters $e_{2}=0.1$, $e_{2}=1, e_{2}=10$, and $e_{2}=100$ are shown in Figure 4 . The condition number 
a

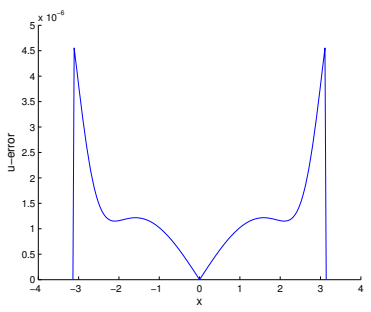

$\mathrm{b}$

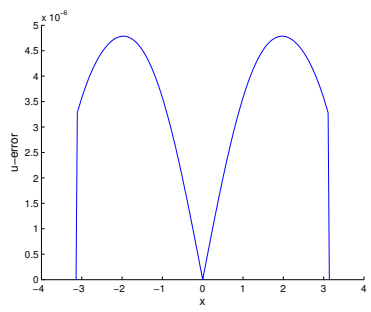

C

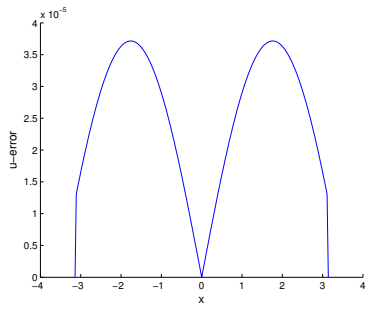

Figure 2. Absolute error graphs of the coupled viscous Burgers' equation (Test problem I) with the uniform set of points; a $(t=0.1)$, b $(t=0.5), \mathrm{c}(t=1)$.

a

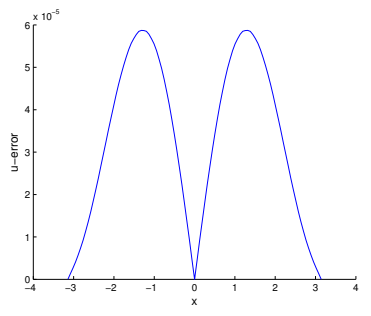

$\mathrm{b}$

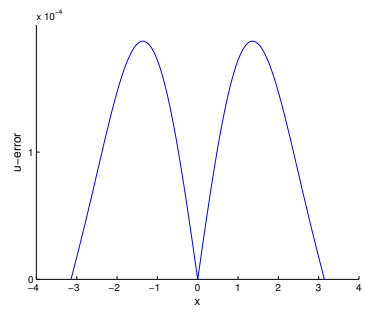

c

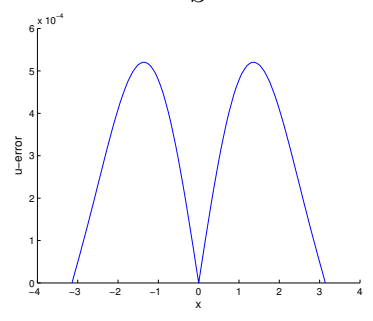

Figure 3. Absolute error graphs of the coupled viscous Burgers' equation (Test problem I) with the Chebyshev set of points; a $(t=0.1)$, b $(t=0.5), \mathrm{c}(t=1)$.

of the matrix $\mathbf{B}_{1}=\mathbf{B}_{2}$ corresponding to those values of $e_{2}$ are also given in Table 4. As shown is Table 4 the condition number increases as the free parameter $e_{2}$ increases. However, large $e_{2}$ gets better accuracy of the method. It seems that having the better accuracy and conditioning can obviously not occur at the same time.

\section{Test problem II)}

In the next experiment, we take $a=-10, b=10$, and $\eta=2$ for different values of $\alpha$ and $\beta$ at time $T=0.5$ and 1 . The exact solution of the equation is given 
Table 2. Spatial rate of convergence for the coupled viscous Burgers' equations (Test problem I), at $T=1$.

\begin{tabular}{llll}
\hline$N_{1}$ & $L_{r}$ & Order & CPU time \\
\hline 2 & $1.330025 E-03$ & - & $2.960 \mathrm{~s}$ \\
4 & $4.392653 E-04$ & 1.598288 & $7.953 \mathrm{~s}$ \\
8 & $1.414223 E-04$ & 1.635083 & $30.64 \mathrm{~s}$ \\
16 & $3.811148 E-05$ & 1.891712 & $239.5 \mathrm{~s}$ \\
32 & $8.989990 E-06$ & 2.083834 & $345.1 \mathrm{~s}$ \\
\hline
\end{tabular}

Table 3. Time rate of convergence for the coupled viscous Burgers' equations (Test problem I), at $T=1$.

\begin{tabular}{llll}
\hline$N_{2}$ & $L_{r}$ & Order & CPU time \\
\hline 2 & $2.000738 E-04$ & - & $4.578 \mathrm{~s}$ \\
4 & $8.567671 E-05$ & 1.223557 & $9.734 \mathrm{~s}$ \\
8 & $2.466290 E-05$ & 1.796561 & $32.89 \mathrm{~s}$ \\
16 & $6.833027 E-06$ & 1.851746 & $247.5 \mathrm{~s}$ \\
32 & $1.851099 E-06$ & 1.884143 & $350.1 \mathrm{~s}$ \\
\hline
\end{tabular}

(a)

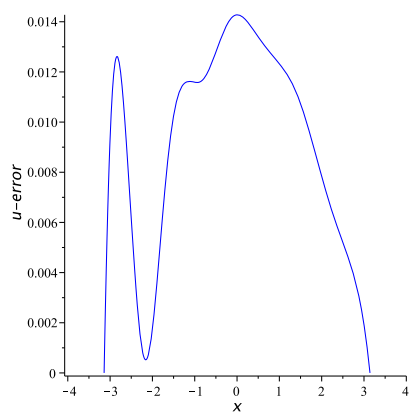

(c)

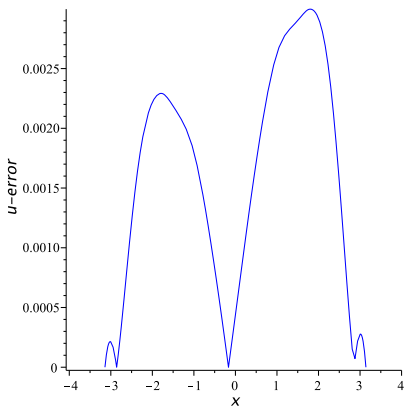

(b)

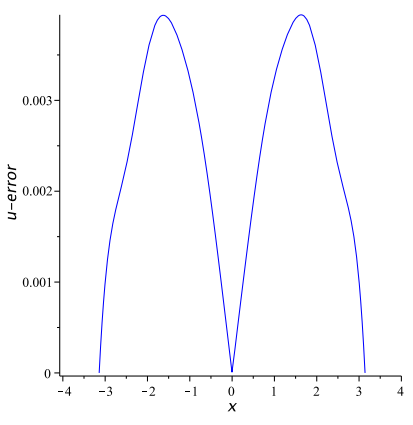

(d)

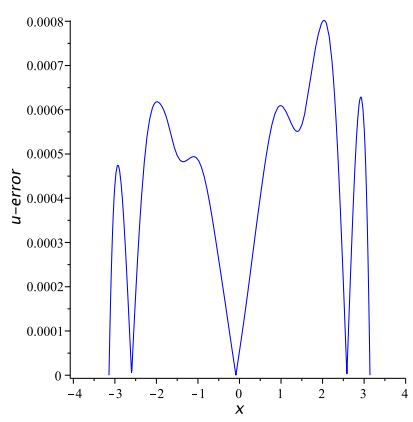

Figure 4. Absolute error graphs of the coupled viscous Burgers' equation (Test problem I); a $\left(e_{2}=0.1\right), \mathrm{b}\left(e_{2}=1\right), \mathrm{c}\left(e_{2}=10\right), \mathrm{d}\left(e_{2}=100\right)$.

by [19] as

$$
\begin{aligned}
& u(x, t)=a_{0}(1-\tanh (A(x-2 A t))), \\
& v(x, t)=a_{0}\left(\left(\frac{2 \beta-1}{2 \alpha-1}\right)-\tanh (A(x-2 A t))\right),
\end{aligned}
$$


Table 4. Condition number of matrix $\mathbf{B}_{1}$ for the coupled viscous Burgers' equations (Test problem I).

\begin{tabular}{ll}
\hline$e_{2}$ & Condition Number \\
\hline 0.1 & $3.8759 E 07$ \\
1 & $1.5761 E 09$ \\
10 & $1.8178 E 10$ \\
100 & $2.5431 E 12$ \\
\hline
\end{tabular}

Table 5. Comparisons of $L_{\infty}$ errors at different time for $u(x, t)$ of Test problem II.

\begin{tabular}{llllllll}
\hline$T$ & $\alpha$ & $\beta$ & $\begin{array}{l}\text { Present Method } \\
(n=5, \\
m=220)\end{array}$ & $\begin{array}{l}{[9]} \\
(h=0.25,\end{array}$ & $\begin{array}{l}{[10]} \\
(h=0.2,\end{array}$ & $\begin{array}{l}{[18]} \\
(h=0.25,\end{array}$ & $\begin{array}{l}{[7]} \\
(h=0.25, \\
\end{array}$ \\
& & & & $\Delta t=0.01)$ & $\Delta t=0.0001)$ & $\Delta t=0.01)$ \\
\hline 0.5 & 0.1 & 0.3 & $4.251 E-05$ & $4.38 E-05$ & $4.167 E-05$ & $9.619 E-04$ & $4.084 E-05$ \\
& 0.3 & 0.03 & $3.698 E-05$ & $4.58 E-05$ & $4.590 E-05$ & $4.310 E-04$ & $4.285 E-05$ \\
1 & 0.1 & 0.3 & $8.150 E-05$ & $8.66 E-05$ & $8.258 E-05$ & $1.153 E-03$ & $8.157 E-05$ \\
& 0.3 & 0.03 & $9.038 E-05$ & $9.16 E-05$ & $9.182 E-05$ & $1.268 E-03$ & $8.873 E-05$ \\
\hline
\end{tabular}

Table 6. Comparisons of $L_{\infty}$ errors at different time for $v(x, t)$ of Test problem II.

\begin{tabular}{llllllll}
\hline$T$ & $\alpha$ & $\beta$ & $\begin{array}{l}\text { Present Method } \\
(n=5,\end{array}$ & $\begin{array}{l}{[9]} \\
(h=0.25,\end{array}$ & $\begin{array}{l}{[10]} \\
(h=0.2,\end{array}$ & $\begin{array}{l}{[18]} \\
(h=0.25,\end{array}$ & $\begin{array}{l}{[7]} \\
(h=0.25, \\
\end{array}$ \\
& & $m=220)$ & $\Delta t=0.01)$ & $\Delta t=0.01)$ & $\Delta t=0.0001)$ & $\Delta t=0.01)$ \\
\hline 0.5 & 0.1 & 0.3 & $4.051 E-05$ & $4.99 E-05$ & $1.480 E-04$ & $3.332 E-04$ & $3.713 E-05$ \\
& 0.3 & 0.03 & $6.341 E-05$ & $1.81 E-04$ & $5.729 E-04$ & $1.148 E-03$ & $7.681 E-05$ \\
1 & 0.1 & 0.3 & $7.158 E-05$ & $9.92 E-05$ & $4.770 E-05$ & $1.162 E-03$ & $7.358 E-05$ \\
& 0.3 & 0.03 & $1.638 E-04$ & $3.62 E-04$ & $3.617 E-04$ & $1.638 E-03$ & $1.572 E-04$ \\
\hline
\end{tabular}

where $a_{0}=0.05, A=\frac{1}{2} a_{0}\left(\frac{4 \alpha \beta-1}{2 \alpha-1}\right)$. The initial and boundary conditions are taken from the exact solution. In our computational work in this case, we put $e_{1}=1, e_{2}=1, b_{1}=1, b_{2}=1, n=5, N_{1}=18$, and $N_{2}=10$. The norm infinity of errors are calculated and compared in Tables 5 and 6 with those available in the literature. It can be noted from Tables 5 and 6 that the results of the proposed method are better than the results of $[9,10,18]$ and in agreement with the results of $[7]$.

\section{Test problem III)}

In the last experiment, we take $a=0, b=1, T=1$, the initial conditions

$$
u(x, 0)=\left\{\begin{array}{ll}
\sin (2 \pi x), & 0 \leq x \leq 0.5, \\
0, & 0.5 \leq x \leq 1,
\end{array} \quad v(x, 0)= \begin{cases}0, & 0 \leq x \leq 0.5, \\
-\sin (2 \pi x), & 0.5 \leq x \leq 1\end{cases}\right.
$$

and zero boundary conditions. To authors knowledge, there is no exact solution available in the literature for this case. The numerical solutions of $u$ and $v$ are depicted graphically in Figures 5 and 6 for $\alpha=\beta=10$ and $\alpha=\beta=100$, respectively, by taking parameters $\eta=2, n=5, N_{1}=18$, and $N_{2}=10$. A sharp decay is noticed in the solutions for the higher values of $\alpha$ and $\beta$. Now, the 

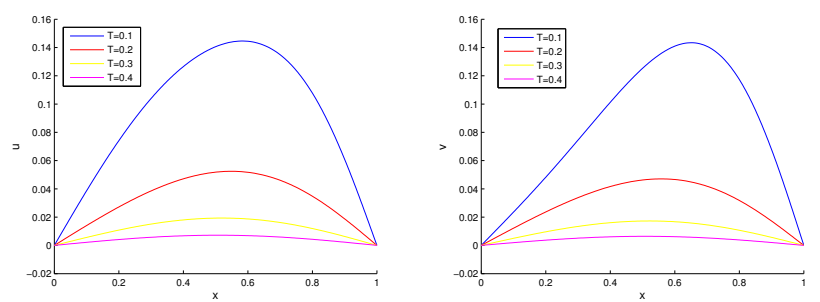

Figure 5. Numerical solutions of the coupled viscous Burgers' equation (Test problem III), for $\alpha=\beta=10, \eta=2$.
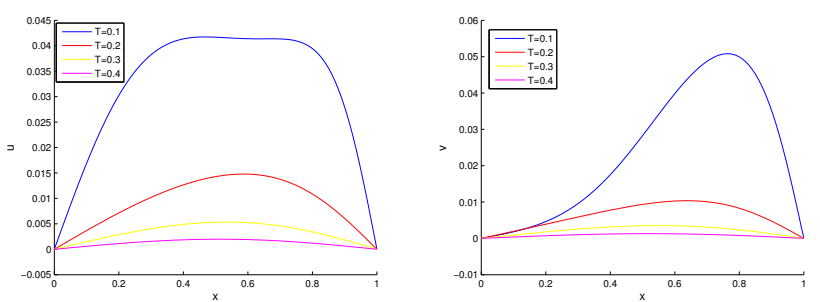

Figure 6. Numerical solutions of the coupled viscous Burgers' equation (Test problem III), for $\alpha=\beta=100, \eta=2$.

(a)

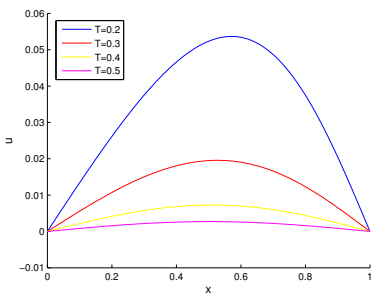

(c)

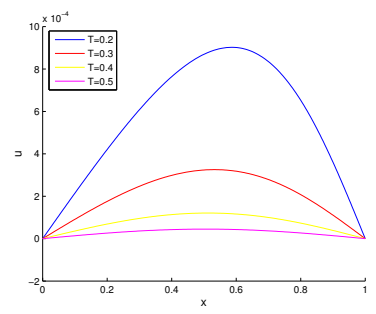

(b)

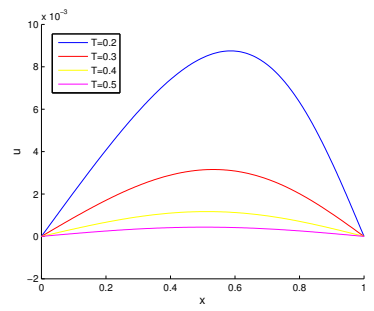

(d)

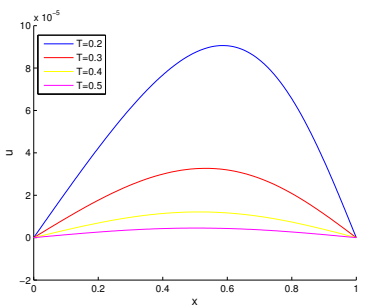

Figure 7. Solution profile of $u$ for the coupled viscous Burgers' equation (Test problem III); a $(\alpha=\beta=10, \eta=20)$, b $(\alpha=\beta=10, \eta=200)$, c $(\alpha=\beta=10, \eta=2000)$, $\mathrm{d}(\alpha=\beta=10, \eta=2000)$.

solution profiles of $u$ are plotted with $\alpha=\beta=10$ for $\eta=20,200,2000,20000$ in Figures $7 \mathrm{a}, 7 \mathrm{~b}, 7 \mathrm{c}$, and $7 \mathrm{~d}$, respectively, in order to show the effect of increasing value of $\eta$. From the figures it can be concluded that the solution decays to zero with increasing time levels and value of $\eta$. It can be also observed that the 

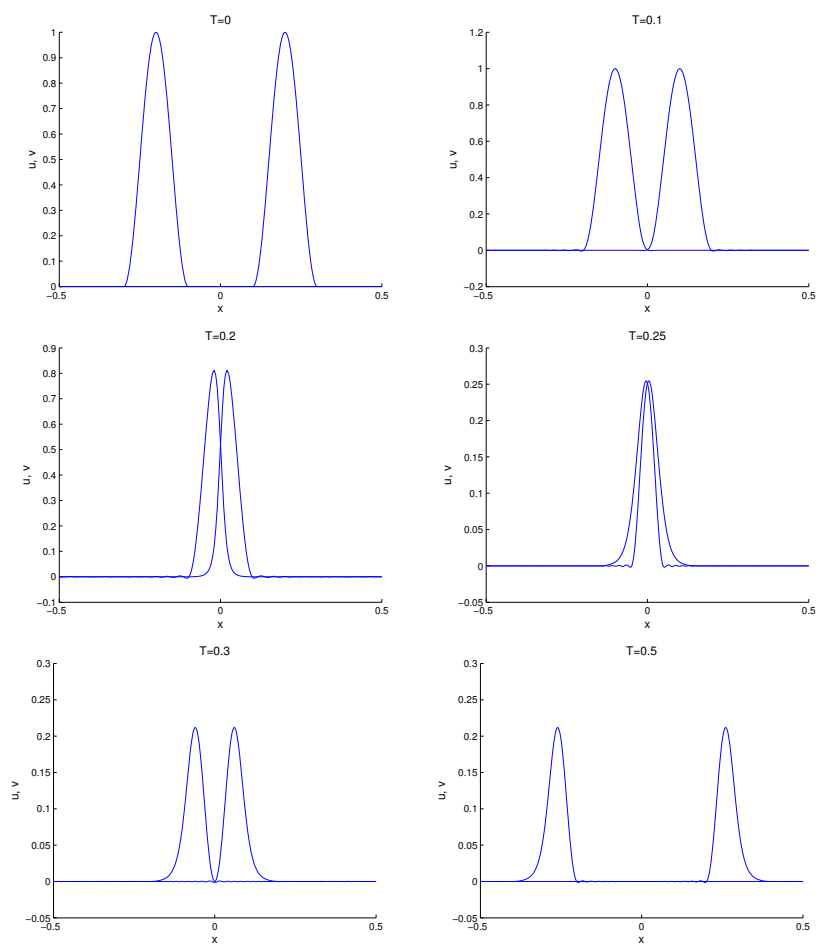

Figure 8. Plots of nonlinear Hyperbolic system.

method is capable in finding the solution at higher values of $\eta$. Our numerical results are in good agreement with the numerical results reported in [10].

\subsection{Nonlinear hyperbolic system}

Consider the nonlinear hyperbolic system

$$
\left\{\begin{array}{l}
u_{t}+u_{x}+\nu u v=0, \\
v_{t}-v_{x}+\nu u v=0,
\end{array} \quad(x, t) \in(a, b) \times(0, T] .\right.
$$

For the sake of comparison, we take $a=-0.5, b=0.5, \nu=100$ with the initial conditions

$$
\begin{aligned}
& u(x, 0)= \begin{cases}0.5[1+\cos (10 \pi x)], & x \in[-0.3,-0.1], \\
0, & \text { otherwise }\end{cases} \\
& v(x, 0)= \begin{cases}0.5[1+\cos (10 \pi x)], & x \in[0.1,0.3] \\
0, & \text { otherwise }\end{cases}
\end{aligned}
$$

and the boundary conditions

$$
u(a, t)=u(b, t)=0 ., \quad v(a, t)=v(b, t)=0 .
$$

The relevant reproducing kernel space $W(\Omega)$ can be defined by $W_{1}^{2}(\Omega)$. The motion and interaction of the waves $u$ and $v$ are depicted in Figure 8 using 
parameters $e_{1}=1, e_{2}=1, b_{1}=1, b_{2}=1, n=5, N_{1}=18$, and $N_{2}=10$. It is clear from Figure 8 that the peaks of the initial solutions $u(x, 0)$ and $v(x, 0)$ are located at $x=-0.2$ and 0.2 , respectively. At $t=0$ these functions are independent of the nonlinear term $u v$. For $t>0$, the nonlinear term $u v$ causes these waves to move without change of shape, $u$ to the right and $v$ to the left. Collision of the two waves occur at $t=0.1$ and $x=0$, which results in change of shapes of the waves. Due to nonlinear interaction the two waves overlap each other near $t=0.25$ and they separate again at $t=0.3$, approximately. From this time onwards the linear term becomes dominant and the pulses lose their symmetry and experience a decrease in the amplitude due to nonlinear interaction as shown in Figure 8. Our numerical results are in good agreement with the numerical results reported in [2] and [7].

\section{Conclusion}

In this work, we proposed an iterative algorithm for solving a class of nonlinear systems of PDEs, on the basis of the reproducing kernel Hilbert space. Results of numerical examples show that the present method is an accurate and reliable analytical-numerical technique. The advantages of the approach lie in the following facts. The method is mesh free, easily implemented and capable in treating various boundary conditions. The method needs no time discretization against $[7,10]$ and any ODE integrator against $[5,9,18]$. Therefore there is no concern about the stability problem and also increasing the end of time $T$ does not increase the CPU time. Also we can evaluate the approximate solution $U_{n, m}(x, t)$ for fixed $n$ and $m$ once, and use it over and over. The approximate solution also converges uniformly to the analytical solution. Unlike the previous studies on the reproducing kernel methods, we avoid having to practise the Gram-Schmidt orthogonalization process. Then the required computational time can be somewhat saved. The main disadvantage of the method is that it can not handle the high dimensional PDEs with irregular regions. Because it needs to work with the tensor product of the reproducing kernel spaces corresponding to each dimension. However, the method [7] uses radial basis functions as kernel functions and hence it does not not have this disadvantage. It seems that the method can be also applied for solving other nonlinear systems of PDEs. We leave this to our further works.

\section{Appendix}

For constructing reproducing kernel $R_{y}(x)$ in $W_{2}[a, b]$, according to (5.1) we have

$$
\begin{aligned}
\left\langle u(x), R_{y}(x)\right\rangle_{W_{2}}= & u^{\prime}(a)\left(e_{1} R_{y}^{\prime}(a)+e_{2} R_{y}^{(4)}(a)\right)+e_{2} u^{\prime \prime}(b) R_{y}^{(3)}(b) \\
& -e_{2} u^{\prime \prime}(a) R_{y}^{(3)}(a)-e_{2} u^{\prime}(b) R_{y}^{(4)}(b)-e_{2} \int_{a}^{b} u(x) R_{y}^{(6)}(x) d x .1
\end{aligned}
$$

Since $R_{y}(x) \in W_{2}[a, b]$, it follows that $R_{y}(a)=0, \quad R_{y}(b)=0$. If

$$
e_{1} R_{y}^{\prime}(a)+e_{2} R_{y}^{(4)}(a)=0, \quad R_{y}^{(3)}(b)=0, \quad R_{y}^{(3)}(a)=0, \quad R_{y}^{(4)}(b)=0,
$$


then (8.1) implies that

$$
\left\langle u(x), R_{y}(x)\right\rangle_{W_{2}}=-e_{2} \int_{a}^{b} u(x) R_{y}^{(6)}(x) d x .
$$

For every $x$ in $[a, b]$, if $R_{y}(x)$ also satisfies

$$
R_{y}^{(6)}(x)=-\frac{1}{e_{2}} \delta(x-y),
$$

then $\left\langle u(x), R_{y}(x)\right\rangle_{W_{2}}=u(y)$. Thus we introduce the analytical representation of $R_{y}(x)$. Consider the following boundary value problem with $y$ as a parameter:

$$
\left\{\begin{array}{l}
R_{y}^{(6)}(x)=0, \quad x \neq y \\
R_{y}(a)=0, \quad R_{y}(b)=0 \\
e_{1} R_{y}^{\prime}(a)+e_{2} R_{y}^{(4)}(a)=0 \\
R_{y}^{(3)}(a)=0, \quad R_{y}^{(3)}(b)=0, \quad R_{y}^{(4)}(b)=0, \\
\left.R_{y}^{(k)}(x)\right|_{x=y^{-0}}=\left.R_{y}^{(k)}(x)\right|_{x=y^{+0}}, \quad k=0,1,2,3,4 \\
\left.R_{y}^{(5)}(x)\right|_{x=y^{+0}}-\left.R_{y}^{(5)}(x)\right|_{x=y^{-0}}=-\frac{1}{e_{2}}
\end{array}\right.
$$

It can be shown that the solution of problem (8.2) is

$$
R_{y}(x)= \begin{cases}c_{1}+c_{2} x+c_{3} x^{2}+c_{4} x^{3}+c_{5} x^{4}+c_{6} x^{5}, & x \leq y \\ d_{1}+d_{2} x+d_{3} x^{2}+d_{4} x^{3}+d_{5} x^{4}+d_{6} x^{5}, & x>y\end{cases}
$$

where the unknown coefficients can be obtained by applying the boundary conditions of (8.2).

\section{References}

[1] N. Aronszajn. Theory of reproducing kernels. Trans. Amer. Math. Soc., 68:337404, 1950. http://dx.doi.org/10.1090/S0002-9947-1950-0051437-7.

[2] R. Chen and Z. Wu. Solving partial differential equation by using multiquadric quasi-interpolation. Appl. Math. Comput., 6:1502-1510, 2007. http://dx.doi.org/10.1016/j.amc.2006.07.160.

[3] M. Cui and Y. Lin. Nonlinear Numerical Analysis in Reproducing Kernel Space. Nova Science Pub. Inc., 2009.

[4] L. Debnath and P. Mikusiński. Hilbert Spaces with Applications. Elsevier Academic Press, USA, 2005.

[5] M. Dehghan and A.Taleei. A Chebyshev pseudospectral multidomain method for the soliton solution of coupled nonlinear Schrödinger equations. Comput. Phys. Comm., 182:2519-2529, 2011. http://dx.doi.org/10.1016/j.cpc.2011.07.009.

[6] F. Geng and X.M. Li. A new method for Riccati differential equations based on reproducing kernel and quasilinearization methods. Abstr. Appl. Anal., 2012. http://dx.doi.org/10.1155/2012/603748. Article ID 603748 
[7] S. Islam, H. Sirajul and U. Marjan. A meshfree interpolation method for the numerical solution of the coupled nonlinear partial differential equations. Eng. Anal. Boundary Elem., 33:399-409, 2009.

http://dx.doi.org/10.1016/j.enganabound.2008.06.005.

[8] D. Kaya. An explicit solution of coupled viscous Burgers' equation by the decomposition method. IJMMS, 11:675-680, 2001.

[9] A.H. Khater, R.S. Temsah and M.S. Hassan. A Chebyshev spectral collocation method for solving Burgers'-type equations. J. Comput. Appl. Math., 222:333350, 2008. http://dx.doi.org/10.1016/j.cam.2007.11.007.

[10] R.C. Mittal and G. Arora. Numerical solution of the coupled viscous Burgers' equation. Commun. Nonlinear Sci. Numer. Simul., 15:1304-1313, 2011. http://dx.doi.org/10.1016/j.cnsns.2010.06.028.

[11] M. Mohammadi and R. Mokhtari. Solving the generalized regularized long wave equation on the basis of a reproducing kernel space. J. Comput. Appl. Math., 235:4003-4014, 2011. http://dx.doi.org/10.1016/j.cam.2011.02.012.

[12] M. Mohammadi and R. Mokhtari. A new algorithm for solving one-dimensional Schrödinger equations in the reproducing kernel space. IJSET-Transaction A., 37:546-523, 2013.

[13] R. Mokhtari and M. Mohammadi. New exact solutions to a class of coupled nonlinearPDEs. Int. J. Nonlinear Sci. Numer. Simul., 10:779-796, 2009. http://dx.doi.org/10.1515/IJNSNS.2009.10.6.779.

[14] R. Mokhtari and M. Mohammadi. Numerical solution of GRLW equation using Sinc-collocation method. Comput. Phys. Comm., 181:1266-1274, 2010. http://dx.doi.org/10.1016/j.cpc.2010.03.015.

[15] R. Mokhtari and M. Mohseni. A meshless method for solving mKdV equation. Comput. Phys. Comm., 183:1259-1268, 2012. http://dx.doi.org/10.1016/j.cpc.2012.02.006.

[16] R. Mokhtari, F. Toutian Isfahani and M. Mohammadi. Solving a class of nonlinear differential-difference equations in the reproducing kernel space. Abstr. Appl. Anal., 2012. Article ID 514103

[17] J. Nee and J. Duan. Limit set of trajectories of the coupled viscous Burgers' equations. Appl. Math. Lett., 11:57-61, 1998. http://dx.doi.org/10.1016/S0893-9659(97)00133-X.

[18] A. Rashid and A. Ismail. A Fourier pseudospectral method for solving coupled viscous Burgers' equations. Comput. Methods Appl. Math., 9:412-420, 2009.

[19] A. Soliman. The modified extended tanh-function method for solving Burgers'type equations. Phys. A, 361:394-404, 2006. http://dx.doi.org/10.1016/j.physa.2005.07.008.

[20] S.M. Wong, Y.C. Hon and M.A. Golberg. Compactly supported radial basis functions for shallow water equations. Appl. Math. Comput., 127:70-101, 2002. http://dx.doi.org/10.1016/S0096-3003(01)00006-6.

[21] L. Yingzhen and Z. Yongfang. Solving nonlinear pseudoparabolic equations with nonlocal boundary conditions in reproducing kernel space. Numer. Algorithms, 52:173-186, 2009. http://dx.doi.org/10.1007/s11075-009-9263-6. 